\title{
Detection of High Frequency Sources in Random/Uncertain Media.
}

\author{
Leon H. Sibul, Christian M. Coviello, Michael J. Roan \\ Applied Research Laboratory \\ The Pennsylvania State University \\ P.O. Box 30 \\ State College, PA. 16804-0030 \\ Lhs2@psu.edu
}

\begin{abstract}
The results of this paper show how randomness and/or uncertainty of medium, boundary conditions, source characterization, and source and receiver motion affects the probability of detection of a narrow band, high frequency source. Using ray acoustic model, we derive expressions for loss of time coherency and its dual, spectral spreading that is caused motion through a medium with random boundary conditions and inhomogeneities. Spectral spreading decreases probability of detection of a narrowband signals. In this analysis both the usual knowledge and the essential uncertainty are incorporated into problem formulation by separating propagation models and boundary conditions into deterministic and random parts. Maximum entropy method (MEM) is used to incorporate essential uncertainty into model. Maximum entropy method uses what is known in its model, but models what is not known with maximum uncertainty. It does not make any unwarranted assumptions about unknown parameters. MEM is used to calculate confidence intervals and mean values of receiver operating characteristics of high-frequency passive and active sonar detectors when signal to noise ratio is a random variable.
\end{abstract}

\section{INTRODUCTION}

The objective of the research that is presented in this paper to investigate how everpresent randomness and uncertainty in propagation medium and source characterization affects active and passive high-frequency sonar signal processing. Motion of sources and receiver through a propagation medium spatially varying sound speed profiles and random boundary conditions is equivalent to signal propagation through a randomly time-varying or stochastic propagation medium that causes a loss of signal coherence, spectral spreading and an increase of entropy (a measure of uncertainty) of the signal. The difficulty of the performance analysis of sonar is further aggravated by uncertainties in source strength, target models, boundary conditions, background noise characterization, and system specifications. All this suggests that sonar performance analysis should reflect essential uncertainties and randomness of the propagation and system parameters, but the analysis should also be based what is known or specified. This can be accomplished by the maximum entropy method (MEM). In this paper we use MEM to derive probability density functions $(P D F)$ that are required to calculate confidence intervals and mean values of receiver operating characteristics (ROC) of simple active and passive detectors that operate in 
random/ uncertain propagation medium. In the case of random medium parameters, the detection statistic is a function of these random medium parameters. Thus, the exceeding of the detection threshold for a given false alarm probability is a random event with a $P D F f_{D}(\theta)$, where $\theta$ is a vector of random parameters. In the simple examples presented in this paper, $\theta$ is a random signal to noise ratio. Randomness of $\theta$ incorporates uncertainty of source strength, propagation loss, and background noise. We feel that plots that show confidence intervals and mean values of the generalized receiver operating characteristics are more effective in displaying the effects of uncertainty and randomness of the medium that so called "range of the day." A sonar operator can use our plots to determine, firstly, what are the confidence intervals for a probability of detection at a specified false alarm probability and, secondly, the mean propagation loss to a given range.

We start with a discussion of modeling of high-frequency propagation in timevarying random media, next we will present a brief review of the maximum entropy method for derivation of probability density functions, then we will present derivation of detection statistics for random parameters, and finally we will calculate confidence intervals for the random receiver operating characteristics. The basic propagation problem that we are considering is a high frequency, multipath propagation with random boundary conditions.

\section{MODELING OF HIGH-FREQUENCY PROPAGATION IN TIME-VARYING RANDOM MEDIA}

We assume that both the source (or target) and receiver are in motion in a propagation medium with a random boundary and an inhomogeneous sound speed profile. Under these conditions the sound propagates through a time-varying, random or stochastic medium. Propagation in stochastic medium can be characterized by stochastic Green's functions [1] or alternatively by random spreading functions [2]. Integral transform pairs relate spreading functions and stochastic Green's functions. Spreading functions are more convenient for analysis of signal processing systems and for derivation of ROCs for both active and passive sonar in stochastic media. Spreading functions are random functions that indicate how a stochastic medium spreads a propagating signal in time and frequency. Hence, spreading functions are time-frequency domain characterizations of narrow band signal propagation and scattering. Wideband spreading functions are wavelet transform domain characterizations of propagation and scattering of wideband signals in stochastic media [3]. Scattering functions, which have been widely used for analysis and synthesis of sonar, radar and communication systems, can be calculated from the spreading functions [1]. The receiver input consists of noise $n(t)$ and signal component or echo $y(t)$ :

$$
y(t)=\iint_{T, \Omega} b(\tau, \omega)[U(\tau, \omega) s(t)] d \tau d \omega,
$$

where $b(\tau, \omega)$ is the spreading function and $U(\tau, \omega)$ is a unitary transformation that transforms the signal $s(t)$ as shown below: 


$$
[U(\tau, \omega) s(t)]=s(t-\tau) \exp (-j \omega t) .
$$

Here $\tau$ is the differential propagation delay and $\omega$ is frequency shift, or Doppler shift in the case of active sonar. According to the ray acoustics [4], discrete multipath propagation can be modeled as a sum of individual time-frequency spread signals:

$$
y(t)=\sum_{i} \iint_{T_{i} \Omega_{i}} b_{i}\left(\tau_{i}, \omega_{i}\right)\left[U\left(\tau_{i}, \omega_{i}\right) s(t)\right] d \tau_{i} d \omega_{i},
$$

In Equation $3 b_{i}\left(\tau_{i}, \omega_{i}\right)$ is the spreading function for the $i-t h$ ray. Thus the time frequency spread signal is a sum of signals that are spread within ray tubes. Under wide-sense stationary, uncorrelated scattering (WSSUS) assumption and under the additional assumption that $s(t)$ is a wide-sense stationary stochastic process, the correlation function of the received signal is

$$
R_{y y}(\tau)=R_{s s}(\tau) \sum_{i} \iint_{\mathrm{T}_{i} \Omega_{i_{i}}} S\left(\tau_{i}, \omega_{i}\right) \exp \left(j \omega_{i} \tau\right) d \tau_{i} d \omega_{i},
$$

where $S\left(\tau_{i}, \omega_{i}\right)$ is the scattering function the is computed by the WSSUS assumption from the spreading functions by Equation 5 , below

$$
S\left(\tau_{i}, \omega_{i}\right) \delta\left(\tau_{i}-\tau_{k}\right) \delta\left(\omega_{i}-\omega_{k}\right)=E\left\{b\left(\tau_{i}, \omega_{i}\right) b^{*}\left(\tau_{k}, \omega_{k}\right)\right\} .
$$

The power spectral density of the received signal is the Fourier transform of the correlation function as expressed by Equation 3:

$$
F_{y}(\omega)=\sum_{i} \iint_{\mathrm{T}_{i} \Omega_{i}} S\left(\tau_{i}, \omega_{i}\right) F_{s}\left(\omega-\omega_{i}\right) d \omega_{i} d \tau_{i} .
$$

Equation 4 shows loss of signal coherency and Equation 6 shows spectral spreading due to multipath effects and motion through inhomogeneous medium with random or rough boundary conditions. Spectral spreading is also an indication of increase of signal uncertainty or entropy

$$
E(y)=-\int_{\Omega} F_{y}(\omega) \ln F_{y}(\omega) d \omega .
$$

In this section we have presented a brief review of characterization of stochastic media by spreading and scattering functions. Spectral spreading, loss of signal coherency and increase of signal uncertainty are all factors that cause decrease of probability of detection. To estimate how probability if detection is affected by medium uncertainty we need for expressions probability density functions. Next we will review the MEM for computing probability density functions that can be used for computation of receiver operating characteristics.

\section{MAXIMUM ENTROPY METHOD FOR PERFORMANCE ANALYSIS OF SIGNAL PROCESSING SYSTEMS IN RANOM MEDIA}

Maximum entropy formalism for continuous random variables maximizes the entropy [5-7]

$$
-\int_{a}^{b} f(x) \ln f(x) d x
$$

subject to the normalization constraint 


$$
\int f(x) d x=1
$$

and moment constraints

$$
\int_{a}^{b} f(x) g_{r}(x) d x=a_{r} \quad r=1,2, \ldots, m .
$$

For constrained maximization, we form the Lagrangian:

$$
L=-\int_{a}^{b} f(x) \ln (x) d x-\left(\lambda_{0}-1\right)\left[\int_{a}^{b} f(x) d x-1\right]-\sum_{r=1}^{m}\left[\int_{a}^{b} f(x) g_{r}(x) d x-a_{r}\right] .
$$

This Lagrangian is in the form of a calculus of variation problem

$$
L=\int_{a}^{b} F\left[x, f(x), f^{\prime}(x)\right] d x
$$

where $F$ is a known function. In this case, the integrand is not a function of $f^{\prime}(x)$ and the Euler-Lagrange equation of the calculus of variations is

which gives

$$
\frac{\partial F}{\partial f(x)}=0
$$

$$
f(x)=\exp \left[-\lambda_{0}-\lambda_{1} g_{1}(x)-\lambda_{2} g_{2}(x)-\cdots-\lambda_{m} g_{m}(x)\right] .
$$

The Lagrange multipliers in the Equation 14 can be determined from the constraint equations

$$
\begin{gathered}
\exp \left(\lambda_{0}\right)=\int_{a}^{b} \exp \left[-\sum_{j=1}^{m} \lambda_{j} g_{j}(x)\right] d x, \\
a_{r} \exp \left(\lambda_{0}\right)=\int_{a}^{b} g_{r}(x) \exp \left[-\sum_{j=1}^{m} \lambda_{j} g_{j}(x)\right] d x \\
\text { and } \\
a_{r}=\frac{\int_{a}^{b} g_{r}(x) \exp \left[-\sum_{j=1}^{m} \lambda_{j} g_{j}(x)\right] d x}{\int_{a}^{b} \exp \left[\sum_{j=1}^{m}-\lambda_{j} g_{j}(x)\right] d x}, \\
r=1,2, \ldots, m .
\end{gathered}
$$

These equations can be used to derive maximum entropy distributions [7]. The maximum entropy distributions that we use in this paper are shown in Table 1 [7]. Specifically, we use a gamma distribution to model random signal to noise ratio, and a beta distribution to model conditional (conditioned on random/uncertain signal to noise ratio) probability of detection. Other well-known maximum entropy distributions include multivariate Gaussian, exponential, truncated exponential, beta of second kind, Laplace and Cauchy distributions. All the relevant information that is required for derivation of these distributions is contained in the moments and 
constraints. Thus, the maximum entropy approach greatly simplifies analysis of signal processing systems in random media. Constraints and moments can be determined from the physics of the media.

TABLE 1. Maximum Entropy Distributions [7]

\begin{tabular}{lcc}
\hline Range & Constraints & Distribution \\
\hline$(-\infty, \infty)$ & $E\{x\}$ and $E\left\{x^{2}\right\}=\sigma^{2}+m^{2}$ & Gaussian: $N\left(m, \sigma^{2}\right)$ \\
{$[0,1]$} & $E\{\ln (x)\}$ and $E\{\ln (1-x)\}$ & $\begin{array}{c}\text { Beta distribution of the first } \\
\text { kind. }\end{array}$ \\
{$[0, \infty)$} & Arithmetic and geometric & Gamma distribution. \\
\hline
\end{tabular}

\section{MATCHED FILTER DETECTION IN RANDOM MEDIA: A SIMPLE EXAMPLE OF MAXIMUM ENTROPY METHOD}

The effects of uncertain target and propagation models, as well as uncertain background noise, manifest in an uncertain signal to noise ratio and time-frequency spreading of the received echo, modeled by Equation 1. Because it is reasonable to assume that, due to multiple scattering random boundaries and propagation through inhomogeneous media, the received signal or echo is a zero mean random process with constrained mean energy. Hence, according to the maximum entropy principle, it is a circularly symmetric (real and imaginary parts have equal variances) complex Gaussian stochastic process. The two hypotheses in this case are

$$
\begin{array}{ll}
H_{1}: & r(t)=\sqrt{E_{r}} \iint_{\mathrm{T} \Omega} b(\tau, \omega)[U(\tau, \omega) s(t)] d \tau d \omega+n(t), \\
H_{0}: & r(t)=n(t),
\end{array}
$$

where $E_{r}$ is expected received energy and $n(t)$ is Gaussian noise, again using maximum entropy assumption. The detection statistic is

$$
\ell(r)=\left|\int_{T} r(t) s^{*}\left(t-\tau_{d}\right) \exp \left(-j \omega_{d}\right) d t\right|^{2} .
$$

The detection statistic $\ell(r)$ is a $\chi_{2}^{2}$ distributed random variable with two degrees of freedom. This follows from the fact that matched filtering operation is a linear operation on a circularly symmetric, complex Gaussian process, $r(t)$; and hence, the magnitude squared of the matched filter output is a sum of squares of the real and imaginary parts [2]. The $p d f s$ of the detection statistics under two hypotheses are simple exponential densities: 


$$
\begin{aligned}
f_{\ell / H_{1}}\left(\ell / H_{1}\right) & =\frac{1}{2 \sigma_{1}^{2}} \exp \left[-\ell / 2 \sigma_{1}^{2}\right] & & \ell \geq 0 \\
& =0, & & \ell<0 \\
f_{\ell / H_{0}}\left(\ell / H_{0}\right) & =\frac{1}{2 \sigma_{n}^{2}} \exp \left[-\ell / 2 \sigma_{n}^{2}\right] & & \ell \geq 0 \\
& =0 & & \ell<0,
\end{aligned}
$$

where $\sigma_{1}^{2}=\sigma_{y}^{2}+\sigma_{n}^{2}$. Conditional probability of detection for a given threshold $\gamma$ for a signal and noise with variance $\sigma_{1}^{2}$ is

$$
\begin{aligned}
f_{D}\left(x / \sigma_{1}^{2}\right) & =\int_{\gamma}^{\infty} \frac{1}{2 \sigma_{1}^{2}} \exp \left[-\ell / 2 \sigma_{1}^{2}\right] d \ell \\
& =\exp \left[-\gamma / 2 \sigma_{1}^{2}\right] .
\end{aligned}
$$

Similarly the probability of false alarm at threshold $\gamma$ and noise variance $\sigma_{n}^{2}$ is

$$
f_{F A}\left(x / \sigma_{n}^{2}\right)=\exp \left[-\gamma / 2 \sigma_{n}^{2}\right] \text {. }
$$

If we fix $f_{F A}$ and solve for the threshold, $\gamma$, we have an expression for the conditional detection $P D F$ in terms of the signal to noise ratio $\theta$ :

$$
f_{D}(x / \theta)=f_{F A}^{\frac{1}{1+\theta}} .
$$

Due to randomness and uncertainty of propagation conditions, target strength and model, we take $\theta$ to be a random variable. It can be shown that a maximum entropy distribution that satisfies these physical constraints is gamma distribution. The marginal probability of detection is:

$$
P_{D}=\int_{\Theta} f_{\Gamma}(\theta) P_{F A}^{\frac{1}{1+\theta}} d \theta .
$$

Equation 22 is used to plot the "mean" of the ROC, however $f_{D}(x / \theta)$ is a function of random/uncertain signal to noise ratio $\theta$. Since $f_{D}(x / \theta)$ is constrained to be in range of $[0,1]$, its possible maximum entropy distribution is beta distribution of first kind with moment constraint

$$
\begin{aligned}
& E\left\{\ln f_{D}(x / \theta)\right\}=\int_{\Theta} f_{\Gamma}(\theta) \frac{\ln P_{F A}}{1+\theta} d \theta=k_{1}, \\
& E\left\{\ln \left(1-f_{D}(x / \theta)\right\}=\int_{\Theta} f_{\Gamma}(\theta) \ln \left[1-\exp \left\{\frac{\ln P_{F A}}{1+\theta}\right\}\right] d \theta=k_{2} .\right.
\end{aligned}
$$


The parameters for the beta distribution are calculated from constraints specified by Equations 23 and 24:

$$
\begin{aligned}
& E\left\{\ln \left(f_{D}(x / \theta)\right)\right\}=\frac{1}{B(m, n)} \int_{0}^{1} x^{m-1}(1-x)^{n-1} \ln (x) d x=f_{1}(m, n), \\
& E\left\{\ln \left(1-f_{D}(x / \theta)\right)\right\}=\frac{1}{B(m, n)} \int_{0}^{1} x^{m-1}(1-x)^{n-1} \ln (1-x) d x=f_{2}(m, n) .
\end{aligned}
$$

The beta density is:

$$
f_{\beta}(x, m, n)=B^{-1}(x, m, n) x^{m-1}(1-x)^{n-1},
$$

where

$$
B(m, n)=\int_{0}^{1} x^{m-1}(1-x)^{n-1} d x
$$

The receiver operating characteristic's mean value and confidence intervals are shown on Figure 1. Parameters for the Gamma density can be computed from mean signal to noise ratio and from the mean of the logarithm of the signal to noise ratio, which in turn can be computed from the sonar equation. Details of this computation will be presented in a longer journal paper.

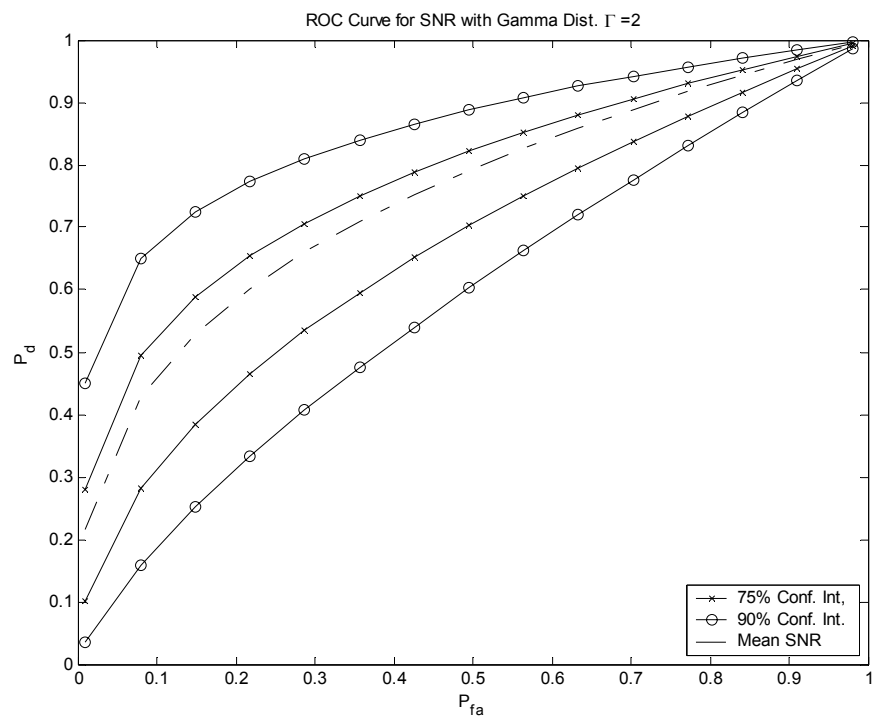

FIGURE 1. Confidence intervals and mean of the receiver operating characteristic of a matched filter detector when signal to noise ratio is a random variable that is Gamma distributed. Gamma $=2$ 


\section{CONCLUDING REMARKS}

High frequency source and receiver motion through inhomogeneous medium with random boundary conditions causes time and frequency spreading of the received signal. This time-frequency spreading can be modeled by spreading functions. In principle, loss of signal coherency, spectral spreading and increase of signal uncertainty can be computed by using the spreading function model. Actual computation requires sophisticated propagation modeling in a media with uncertain and frequently unspecified parameters. In this paper, we presented an example of application of maximum entropy method (MEM) to the performance analysis of a simple matched filter detector. This analysis can also applied to passive sonar detectors. MEM is a constrained optimization problem that maximizes entropy using known moments and range of the random variable as constraints. MEM is maximally uncertain in what is not warranted by data or known models [5-7]. In this performance analysis, uncertainty of propagation and background noise was accounted by treating the received signal to noise ratio as a random variable whose $P D F$ is calculated according to the maximum entropy principle. According to the maximum entropy principle, the signal to noise ratio is a Gamma distribution and the conditional probability of detection is a beta distribution of second kind. Parameters of the Gamma distribution can be determined from the mean and logarithmic mean of the signal to noise ratio. The simple example of application of MEM that has been presented in this paper can be extended to more complicated problems.

\section{ACKNOWLEDGEMENTS}

This material is based on the work supported by the Office of Naval Research under Contract No.N00024-02-D-6604. Dr. John Tague has suggested this problem.

\section{REFERENCES}

1. Adomian, G., "Linear Random Operator Equations in Mathematical Physics I, II III," Jour. Math.Phys., 11, 1069-1084 (1970), 12, 1944-1655, (1971).

2. Van Trees, H.L.,, Detection, Estimation and Modulation Theory, Wiley, New York, 1971, Chapters 9-13.

3. Sibul, L.H., Weiss, L.G., and Dixon, T.L., "Characterization of Stochastic Propagation and Scattering via Gabor and Wavelet Transforms," Jour. Comp. Acoustics, 2, 345-369 (1994)

4. Jensen, FB., Kuperman, W.A., Porter, M.B., and Smith, H., Computational Ocean Acoustics, AIP Press, New York, 1994, pp. 454-459.

5. Jaynes, E.T., "On the Rational of Maximum-Entropy Method," Proc. IEEE, 70, 939-952 (1982).

6. Jaynes, E.T., "Prior Probabilities," IEEE Trans. on System. Sciences and Cybernetics, 4, 227-247, (1968).

7. Kapur, J.N. and Kesavan, H.K., Entropy Optimization, Principles with Applications, Academic Press, Boston, 1992. 